of about $1 \cdot 3$, and then gradually falls and attains the value of unity at a distance of about $1,440 \mathrm{~m}$. Beyond this, the value becomes less than unity and decreases gradually with the distance.

The negative attenuation, as has been pointed out by Ratcliffe and White ${ }^{1}$, is to be expected under certain conditions from Sommerfeld's ${ }^{2}$ theory of attenuation. According to Rolf ${ }^{3}$, Sommerfeld's expression for attenuation can be represented in terms of only two quantities $q$ and $b$ when the field on the surface of the earth is considered. The quantities involve the electrical conductivity $\sigma$, the dielectric constant $\varepsilon$ of the earth, the wave-length $\lambda$ of the wireless wave, and the distance $d$ from the trans. mitter.

Rolf has shown

$$
\tan b=\frac{\varepsilon+1}{6 \lambda \sigma} \cdot 10^{-15} \text { and } q=\frac{2 \pi \sin b}{(\varepsilon+1) \lambda} \cdot d
$$

where distances are expressed in kilometres, $\varepsilon$ in E.S.U. and $\sigma$ in E.M.U. The attenuation curves drawn by Rolf show that for certain values of $b$ there is negative attenuation.

Making use of Rolf's graphs and Sommerfeld's pormula in the form given by Rolf and comparing the theoretical values of attenuation with our observed values, we have been able to determine the values of $b, \varepsilon$ and $\sigma$ in the two directions for $\lambda=160 \mathrm{~m}$. In direction $A$, we find that, up to about $3,500 \mathrm{~m}$., there is agreement between theory and experiment for $b=60^{\circ}, \varepsilon=14$ E.S.U. and $\sigma=0.9 \times 10^{-14}$ E.M.U. Beyond this, in the same direction towards the river side we require, $b=30^{\circ}, \varepsilon=26 \quad$ E.S.U. and $\sigma=4.8 \times 10^{-14}$ E.M.U. for good agreement with Sommerfeld's theory. From the comparatively larger moisture-content of the soil, the lower value of $b$ and the higher values of $\varepsilon$ and $\sigma$ are to be expected in this region. For direction $B$ we have to put $b=40^{\circ}, \varepsilon=9$ E.S.U. and $\sigma=1 \cdot 24 \times 10^{-14}$ E.M.U. to suit Sommerfeld's theory. The theoretical curves are shown in Fig. 1 along with the experimental values of the attenuation. The observed value of attenuation at distance $d=710 \mathrm{~m}$. in direction $A$ is a little too low. This is attributed to the slight screening action of a brick wall.

The values of $\varepsilon$ and $\sigma$ obtained from the attenuation measurements for $\lambda=160 \mathrm{~m}$. agree well with the values obtained by us for the different specimens of Dacea soil by the direct laboratory method.

It should be mentioned that the negative attenuation was first observed by Ratcliffe and Barnett employing wireless waves of $1,600 \mathrm{~m}$. wave-length from the Daventry Station of the B.B.C. Ratcliffe and Shaw ${ }^{5}$ also observed the same thing with a shorter wave-length $(30 \mathrm{~m}$.).

Very recently, Norton ${ }^{6}$ has pointed out an error in Rolf's calculations. Some calculations which we have made lead us to suppose that this error does not materially alter the attenuation curves given by Rolf, at least for very short distances from the trans. mitter.

$$
\begin{aligned}
& \text { D. N. Chandhuri. } \\
& \text { Physies Laboratory, } \\
& \text { Dacca University. } \\
& \text { Aug. } 19 . \\
& 1 \text { Ratcliffe and White, Nature, 125, 926; } 1930 . \\
& \text { Sommerfeld, Ann. Phys., 4, 28, 665; } 1909 . \\
& 3 \text { Rolf, Proc. I.R.E., 18, Ňo. 3, March } 1930 . \\
& \text { - Ratcliffe and Barnett, Proc. Camb. Phil. Soc., 23, } 288 . \\
& \text { Ratcliffe and Barnett, Proc. Camb. Phil. Soc., } \\
& \begin{array}{l}
\text { Ratcliffe and Shaw, NATURE, 124, } \\
\text { Norton, NATURE, 135, 955; } 1935 .
\end{array}
\end{aligned}
$$$$
\text { S. R. Khastgir. }
$$

\section{Dissociation Energy of Carbon Monoxide and the Heat of Sublimation of Carbon}

In two letters, published in Nature of June 29, P. Goldfinger, W. Lasareff and B. Rosen have calculated the heat of dissociation of carbon monoxide and afterwards the heat of sublimation of carbon. They obtain their figures from the predissociations of the term $B^{1} \Sigma$ and the number of observed vibrational levels of the term $A^{1} \Pi$ of CO. There is, however, still another possibility of determining $D_{(\mathrm{CO})}$ originally suggested by Kaplan ${ }^{1}$. Of each of the two electronic states $b^{3} \Sigma$ and $b^{\prime}$, only one vibrational level is known, and this leads to the assumption of a repulsive curve, intersecting the potential curves of these two levels at 10.6 v.e. This value is well between the lower and upper limits $(10.4$ and 11.07 v.e. respectively) calculated by Goldfinger and Lasareff, but slightly below their final estimated value of $11 \cdot 0 \pm 0 \cdot 1$ v.e.

The repulsive curve which intersects at $10 \cdot 6$ v.e. is then the lowest one of all those which involve the level $C\left({ }^{3} P\right)+O\left({ }^{3} P\right)$ of the separated system, and in an earlier publication ${ }^{2}$ we have, therefore, taken the slightly lower value of 10.45 v.e. as that of $D_{(\mathrm{CO})}$ which leads to a value of $155.7 \mathrm{kcal} . / \mathrm{mol}$. for the heat of sublimation of carbon ( $\beta$-graphite) at $25^{\circ} \mathrm{C}$.

The value of 10.45 v.e. for $D_{(\mathrm{CO})}$ may be confirmed in the following way. The level $B^{1} \Sigma$ lies at 10.72 v.e.; linear extrapolation yields a value of its energy of dissociation of 2.94 v.e., which is not necessarily correct, but is certainly an upper limit of it. This value is based on the value of the enharmonic constant of $50 \mathrm{~cm} .^{-1}$, which is not certain ; it appears that this value is the lower limit for the enharmonic constant, the other possible value being $76 \mathrm{~cm}^{-1}$. Since this level cannot be correlated to the level $\mathrm{C}\left({ }^{3} P\right)+\mathrm{O}\left({ }^{3} P\right)$, and since it certainly involves a level of the separated system with equal multiplicity of the two atoms, it has to be correlated to the level $\mathrm{C}\left({ }^{1} D\right)+\mathrm{O}\left({ }^{1} D\right)$. The two energies of excitation of $C$ and $O$ are together 3.21 v.e., giving an upper limit of $D_{(\mathrm{CO})}$ of $10 \cdot 72+2 \cdot 94-3 \cdot 21=10 \cdot 45 \mathrm{v}$.e.

H. Lessheim.

Muslim University, Aligarh.

'J. Kaplan, Phys. Rev., 37, 1406 ; 1931.

2 H. Lessheim and R. Samuel, Proc. Phys. Soc. Lond., 46, $523 ; 1934$.

\section{Oxygen in the Sun's Chromosphere}

Photographs which I have taken at the Kodaikanal Observatory show that oxygen is a normal constituent of the sun's chromosphere, and is probably in great abundance.

Placing the slit of a spectrograph tangentially to the image of the sun's limb, I have found that the infra-red triplet of oxygen, $\lambda \lambda 7771,7774$ and 7775 , shows as emission lines in the sun's chromosphere. This is not a unique appearance in one photograph only, for the reversal of the oxygen triplet into bright lines has been photographed every day since it was first observed.

Fig. 1 is a microphotometric record of one of my photographs taken on August 23, and clearly shows the oxygen lines as emission lines brighter than the continuous spectrum from the sky, compared with the neighbouring $\mathrm{Fe}$ and $\mathrm{Ni}$ absorption lines in the sky spectrum. When the chromosphere is not on 